\title{
Multinodular Goiter as the Initial Presentation of Systemic Sarcoidosis: Limitation of Fine-Needle Biopsy
}

\author{
Thanh D Hoang DO, Vinh Q Mai DO, Patrick W Clyde MD, \\ Babette C Glister MD, and Mohamed KM Shakir MD
}

\begin{abstract}
Sarcoidosis is a chronic systemic disease characterized by noncaseating granulomas. Thyroid involvement is rare, with a prevalence of $1-4 \%$ in large series of autopsied patients with systemic sarcoid. We report a case of a 65 -year-old woman with a nontoxic multinodular goiter, dyspnea in the supine position, and rightward tracheal deviation as the initial presentation of systemic sarcoidosis. Fine-needle biopsy of the thyroid mass was consistent with benign adenomatoid goiter. A total thyroidectomy was performed because of compression symptoms. Histopathology revealed numerous diffuse noncaseating granulomata typical of thyroid sarcoidosis. Subsequent chest computed tomography showed extensive bulky mediastinal and hilar adenopathy and multiple small pulmonary nodules consistent with sarcoidosis. Transbronchial fine-needle biopsy also revealed noncaseating granulomas. The patient's supine dyspnea resolved quickly after total thyroidectomy. This case report illustrates that in patients with known sarcoidosis, careful thyroid examination is essential and supine dyspnea may be directly related to tracheal compression by a multinodular goiter rather than pulmonary sarcoidosis. Key words: thyroid sarcoidosis; noncaseating granuloma; multinodular goiter; sarcoidosis. [Respir Care 2011;56(7):1029-1032]
\end{abstract}

\section{Introduction}

Sarcoidosis is a systemic noncaseating granulomatous disorder that involves various organs, including the lymph nodes, lungs, eyes, liver, spleen, and skin. Sarcoid granuloma within the thyroid gland is rare. The first reported case was in 1938, in which Spencer and Warren described a patient with generalized sarcoidosis and postmortem discovery of thyroid involvement. ${ }^{1}$ Thyroid sarcoidosis incidence was $1-4 \%$ in large series of autopsied patients with systemic sarcoid. ${ }^{2,3}$ We report a patient with nontoxic multinodular goiter as the initial presentation of systemic sarcoidosis.

The authors are affiliated with the Department of Endocrinology, National Naval Medical Center, Bethesda, Maryland.

The authors have disclosed no conflicts of interest. The views expressed in this article are those of the authors and do not necessarily reflect the official policy or position of the Department of the Navy, Department of Defense, or the United States Government.

Correspondence: Thanh D Hoang DO, Department of Endocrinology, National Naval Medical Center, 8901 Wisconsin Ave, Bethesda MD 20889-5600. E-mail: thanh.hoang@med.navy.mil.

DOI: $10.4187 /$ respcare .01000

\section{Case Report}

A 65-year-old woman presented for evaluation of multiple thyroid nodules discovered during a routine physical examination. Chest radiograph initially showed rightward tracheal deviation in the neck, but without mediastinal or parenchymal abnormality. Thyroid ultrasound revealed an enlarged multinodular goiter with a dominant $3.4 \times 2.5 \times 3.8 \mathrm{~cm}$ ill-defined mass in the left lobe. The right and left lobes measured $2.3 \times 2.1 \times 5.4 \mathrm{~cm}$ and $3.7 \times 3.9 \times 8.5 \mathrm{~cm}$, respectively. The patient denied dysphagia, odynophagia, chest pain, or dyspnea on exertion, but reported some pressure and dyspnea in the supine position. She also had a history of palpitation that improved with $\beta$ antagonist therapy. Her medical history included thalassemia minor and hypertriglyceridemia. Her medications included gemfibrozil $600 \mathrm{mg}$ twice daily and folic acid $1 \mathrm{mg}$ daily. She denied tobacco or alcohol use. Blood pressure was 133/77 mm Hg, heart rate was 98 beats/ min, and respiratory rate was 16 breaths/min. Physical examination revealed an enlarged nontender thyroid gland of approximately $35 \mathrm{~g}$, without cervical or supraclavicular lymphadenopathy. Her lungs were clear to auscultation, without wheezing, rales, or rhonchi. The cardiac and neu- 


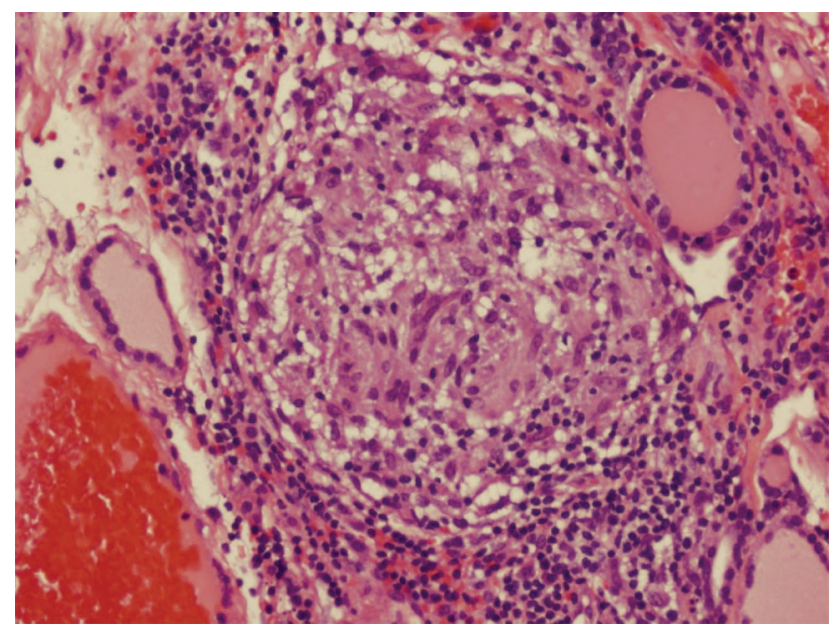

Fig. 1. Thyroid biopsy shows numerous diffuse noncaseating granulomata involving both the thyroid lobes.

romuscular examinations were normal. Thyroid function test showed thyroid stimulating hormone (thyrotropin) $0.672 \mathrm{mcIU} / \mathrm{mL}$ (reference $0.35-5.5 \mathrm{mcIU} / \mathrm{mL}$ ) and free T4 (free thyroxine) $1.30 \mathrm{ng} / \mathrm{dL}$ (reference $0.89-1.76 \mathrm{ng} / \mathrm{dL}$ ). Her erythrocyte sedimentation rate was $19 \mathrm{~mm} / \mathrm{h}$ (reference $4-27 \mathrm{~mm} / \mathrm{h}$ ). Thyroid uptake and scan were not indicated because she was clinically and biochemically euthyroid. Fine-needle biopsy of the thyroid mass showed cellular changes consistent with benign adenomatoid goiter. Because of the compression symptoms and to exclude malignancy, she underwent total thyroidectomy. Although preoperative spirometry was not performed, the evidence of tracheal deviation and the supine dyspnea suggested extrathoracic obstruction.

Intraoperatively her thyroid was soft, diffusely enlarged (left lobe greater than right lobe), and nodular. There were no grossly enlarged central lymph nodes in the neck. The thyroidectomy was without complications and she was discharged to home the day after surgery, with a normal serum calcium level. The supine dyspnea quickly resolved after thyroidectomy. Histopathology of the thyroid specimen revealed numerous diffuse noncaseating granulomata involving both the thyroid lobes (Fig. 1), which prompted further systemic evaluation for sarcoidosis. Grocott methenamine silver and Ziehl-Neelsen stains were negative for fungi and acid-fast bacilli, respectively. Tuberculin skin test was negative. Computed tomogram showed extensive bulky mediastinal and hilar adenopathy and multiple small pulmonary nodules consistent with pulmonary sarcoidosis (Fig. 2). Transbronchial fine-needle biopsy of the right paratracheal lymph node revealed noncaseating granulomas. Pulmonary function tests showed normal baseline spirometry and diffusing capacity for carbon monoxide. Echocardiography showed normal left-ventricle wall motion, left-ventricular ejection fraction of 55-60\%, and nor-
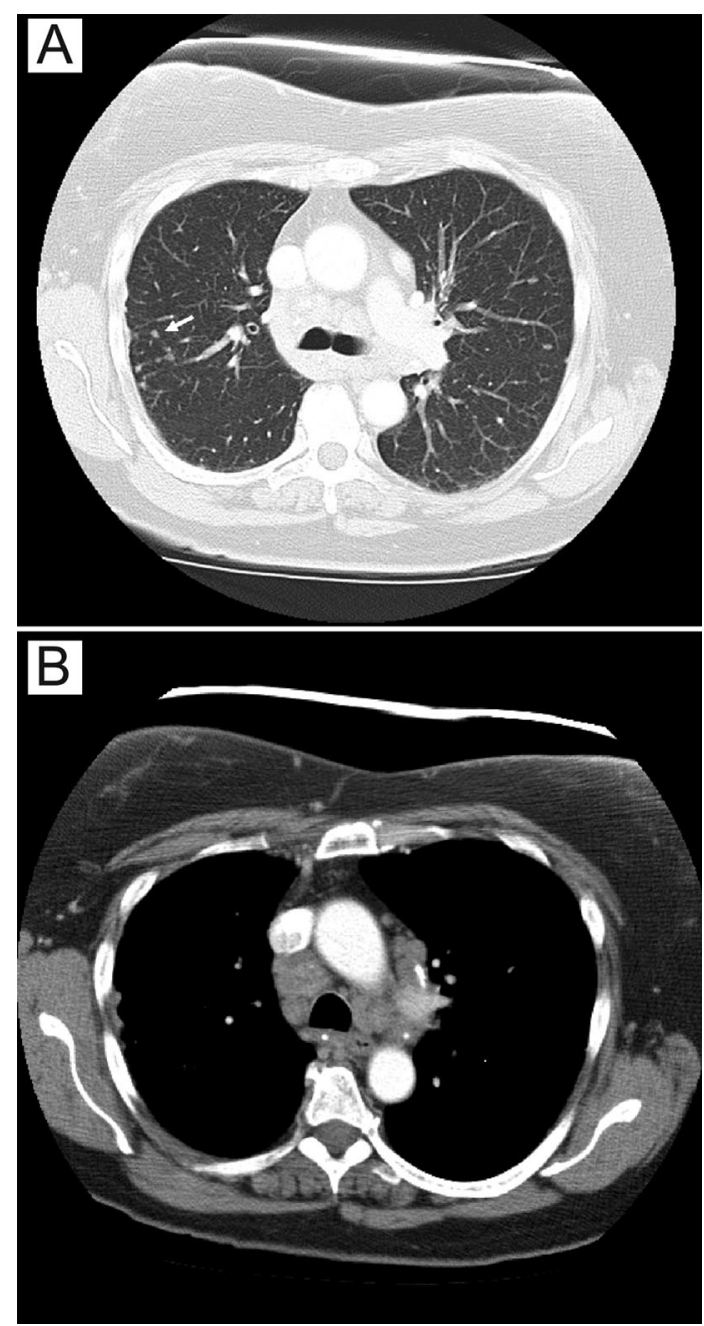

Fig. 2. Computed tomogram shows multiple small pulmonary nodules (A) and extensive bulky mediastinal and hilar adenopathy $(B)$ consistent with pulmonary sarcoidosis.

mal valvular morphology and function. Cardiac magnetic resonance imaging revealed normal T2-weighted myocardial delayed enhancement, without evidence of infiltrative cardiomyopathy or cardiac sarcoid. Table 1 shows the laboratory results.

\section{Discussion}

The diagnosis of sarcoidosis is based on the exclusion of other known etiologies of granulomatous pathology. Sarcoidosis can involve many organs without necessarily producing any signs or symptoms. The most frequent sites of involvement are the lungs, the mediastinal and superficial lymph nodes, and the skin. ${ }^{2}$ Involvement of the thyroid by sarcoidosis is rare, as evidenced by sporadic case reports. ${ }^{4}$ Thyroid sarcoidosis has been associated with Hashimoto disease, Graves disease, thyrotoxicosis, papil- 
Table 1. Laboratory Values at Initial Diagnosis

\begin{tabular}{lcc}
\hline \hline & $\begin{array}{c}\text { Measured } \\
\text { Value }\end{array}$ & $\begin{array}{c}\text { Reference } \\
\text { Range }\end{array}$ \\
\hline Serum calcium (mg/dL) & 10.0 & $8.7-10.4$ \\
Ionized calcium (mmol/L) & 1.23 & $1.12-1.32$ \\
Albumin (g/dL) & 4.9 & $3.4-5.0$ \\
1,25 dihydroxy-vitamin D (pg/mL) & 66 & $18-72$ \\
25 hydroxy-vitamin D (ng/mL) & 37 & $30-80$ \\
Serum parathyroid hormone (pg/mL) & 46 & $13-75$ \\
Angiotensin converting enzyme (U/L) & 30 & $9-67$ \\
Thyroid stimulating hormone (thyrotropin) & 2.1 & $0.4-5.5$ \\
$\quad$ (mcIU/mL) & 1.46 & $0.89-1.76$ \\
Free T4 (ng/dL) & 107 & $0-80$ \\
Thyroglobulin Ab (IU/mL) & 10 & $0-20$ \\
Thyroperoxidase Ab (IU/mL) & & \\
\hline
\end{tabular}

lary thyroid carcinoma, nodular goiter, and painful thyroid nodules. ${ }^{5}$ Thyroid involvement has seldom been reported as the first manifestation of the disease. ${ }^{6}$ Noncaseating granulomas are not specific for sarcoidosis and may be seen in tuberculosis, atypical mycobacterial infections, fungal infections, brucellosis, and Hodgkin disease. Various histological features, such as multinucleated giant cells, epithelioid cell granulomas, and lymphocytic infiltration, can also be present in other infectious and noninfectious diseases of the thyroid. ${ }^{7}$ De Quervain thyroiditis is another granulomatous cause of thyroid involvement, but we excluded it because the patient did not have fever, malaise, thyrotoxicosis associated with a painful or tender thyroid, increased erythrocyte sedimentation rate, or leukocytosis. Additionally, the histopathology findings were not consistent with De Quervain thyroiditis. Supine dyspnea was the presenting symptom in our patient, and the imaging studies excluded cardiac involvement.

The serum calcium and 1,25-dihydroxy vitamin D levels were both normal, probably due to the early stage of her sarcoidosis, because abnormalities in calcium metabolism usually occur in patients with active sarcoidosis and large total-body burden of granulomas. ${ }^{8}$ Furthermore, though thyroid function test and anti-thyroperoxidase antibody level were normal, anti-thyroglobulin antibody level was minimally elevated. This may suggest coincidental finding of an underlying autoimmune process, such as Hashimoto thyroiditis, since it is common in the general population. ${ }^{9}$ Previous reports also indicated that thyroid autoantibodies are relatively common in sarcoidosis. ${ }^{10,11}$ However, the thyroid histopathology from our patient found no evidence of Hashimoto thyroiditis. Possibilities of immunoregulatory defects have been postulated, but the pathogenesis of sarcoidosis and Hashimoto thyroiditis remains unclear. ${ }^{11}$

Although thyroid fine-needle biopsy may aid in early diagnosis and management of thyroid nodular diseases, with an accuracy of $80-97 \%$, most cases of thyroid sarcoidosis have been diagnosed either postoperatively, or at autopsy, or when other manifestations of sarcoidosis appear. ${ }^{12-14}$ Ozkan et al reported that less than $1 \%$ of patients with cold thyroid nodules had noncaseating granulomas. ${ }^{5}$ In our patient, the supine dyspnea quickly resolved after total thyroidectomy, which suggests that multinodular goiter was responsible for her compression respiratory symptoms. She did not have any other systemic complaints of sarcoidosis. The inability of fine-needle biopsy to diagnose sarcoidosis in this case is consistent with the findings of Warshawsky et al. ${ }^{14}$ These lesions might be missed because of the scattered nature of sarcoid infiltrations in the thyroid gland. ${ }^{15}$ However, the histopathology of the resected thyroid gland confirmed sarcoidosis, which led to further investigation for systemic involvement, which was confirmed via computed tomography. Warshawsky et al reported resolution of a palpable thyroid nodule after glucocorticoid therapy in a patient with systemic sarcoidosis, ${ }^{14}$ whereas our patient underwent total thyroidectomy before the diagnosis of sarcoidosis.

This case illustrates that nontoxic multinodular goiter can be the initial presentation of sarcoidosis, and the diagnosis of sarcoidosis can be easily missed. When histology reveals noncaseating granulomata, further evaluation for systemic sarcoidosis, including plain radiographs and computed tomography, should be obtained. In patients with known systemic sarcoidosis, careful thyroid palpation and thyroid function tests should be performed to evaluate for thyroid involvement. Additionally, in patients with pulmonary sarcoidosis and supine dyspnea, a multinodular goiter due to sarcoidosis may be responsible. Further studies are needed to elucidate the accuracy and utility of fine-needle biopsy in diagnosing sarcoid involvement of the thyroid, the effects of glucocorticoid therapy on the resolution of sarcoid thyroid nodules, and the possible cause-and-effect relationship between systemic sarcoidosis and thyroid disorders.

\section{REFERENCES}

1. Spencer J, Warren S. Boeck's sarcoid: report of a case with clinical diagnosis confirmed at autopsy. Arch Intern Med 1938;62(2):285-296.

2. Vailati A, Marena C, Aristia L, Sozze E, Barosi G, Inglese V, et al. Sarcoidosis of the thyroid: report of a case and a review of the literature. Sarcoidosis 1993;10(1):66-68.

3. Mayock RL, Bertrand P, Morrison CE. Manifestations of sarcoidosis. Analysis of 145 patients with a review of nine series selected from the literature. Am J Med 1963;35(1):67-89.

4. Cilley RE, Thompson NW, Lloyd RV, Shapiro B. Sarcoidosis of the thyroid presenting as a painful nodule. Thyroidology 1988;1(1):61-62.

5. Ozkan Z, Oncel M, Kurt N, Kargi AB, Ozdemir N, Kaptanoglu L, et al. Sarcoidosis presenting as cold thyroid nodules: report of two cases. Surg Today 2005;35(9):770-773.

6. Weiss IA, Limaye A, Tchertkoff V, Brener JL. Sarcoidosis of the thyroid clinically mimicking malignancy. NY State J Med 1989; 89(10):578-580. 


\section{Multinodular Goiter as the Initial Presentation of Systemic Sarcoidosis}

7. Harach HR, Williams ED. The pathology of granulomatous diseases of the thyroid gland. Sarcoidosis 1990;7(1):19-27.

8. Bell NH. Endocrine complications of sarcoidosis. Endocrinol Metab Clin North Am 1991;20(3):645-654.

9. Dayan CM, Daniels GH. Chronic autoimmune thyroiditis. N Engl J Med 1996;335(2):99-108.

10. Singer P. Primary hypothyroidism due to other causes. In: Braverman LE, Utiger RD, editors. Werner \& Ingbar's the thyroid: a fundamental and clinical text, eighth edition. Philadephia: Lippincott, Williams \& Wilkins; 2000:758.

11. Rubinstein I, Baum GL, Hiss Y, Margaliot S, Yellin A. Sarcoidosis and Hashimoto's thyroiditis: a chance occurrence? Respiration 1985; 48(2):136-139.
12. Hamming JF, Goslings BM, Steenis GJ, Claasen HR, Hermans J, Velde CJ. The value of fine needle aspiration biopsy in patients with nodular thyroid disease divided into groups of suspicion of malignant neoplasms on clinical grounds. Arch Intern Med 1990;150(1):113-116.

13. Ricker W, Clark M. Sarcoidosis. A clinico-pathologic review of 300 cases, including 22 autopsies. Am J Clin Pathol 1949;19(8): 725-749.

14. Warshawsky ME, Shanies HM, Rozo A. Sarcoidosis involving the thyroid and pleura. Sarcoidosis Vasc Diffuse Lung Diseases 1997; 14(2):165-168

15. Yarman S, Kahraman H, Tanakol R, Kapran Y. Concomitant association of thyroid sarcoidosis and Graves' disease. Horm Res 2003; 59(1):43-46. 